\title{
Lesión de plexo braquial obstétrico; una alternativa de manejo quirúrgico. Reporte de un caso y revisión de la literatura
}

\author{
Obstetrical brachial plexus injury; an alternative of surgery \\ management. Case report and literature review
}

\begin{abstract}
Leonardo López Almejo, ${ }^{*}$ Francisco Yamil Pérez Lima,${ }^{\ddagger}$ Marlene Vanessa Salcido Reyna ${ }^{\S}$
*Cirujano Ortopedista UNAM, Cirujano de Mano UAG, Profesor Titular del Postgrado en Cirugía de Plexo Braquial y Nervio Periférico. Universidad Autónoma de Coahuila. Máster en Cirugía de Plexo Braquial, Clínica Mielina Aguascalientes; ¥ Cirujano Ortopedista. Máster en Cirugía de Plexo Braquial y Nervio Periférico. BPIS. Cirujano de Mano. Hospital General de Mexicali; §Cirujano Ortopedista, Diplomado en Cirugía de Plexo Braquial. Hospital General de Mexicali.
\end{abstract}

\begin{abstract}
Resumen
En la actualidad, el manejo quirúrgico de las lesiones del plexo braquial constituye un gran reto. A pesar de las medidas preventivas aplicadas por los sistemas de salud, las lesiones del plexo braquial continúan presentándose con una incidencia de 0.38 a 5.1 por 1,000 nacimientos vivos a nivel mundial y en México es de 0.37 a 0.87 por cada 1,000 nacimientos vivos. Cabe señalar que $20 \%$ de estos pacientes tendrán un déficit neurológico residual que impactará directamente la función de la extremidad superior. El reto de un tratamiento quirúrgico es llevar al paciente a la mayor funcionalidad posible sin deteriorar la función residual que pueda existir. La decisión quirúrgica es determinada por el cirujano al considerar las manifestaciones clínicas, la ausencia de recuperación neurológica en el transcurso del tiempo y los hallazgos quirúrgicos. Lamentablemente en muchas ocasiones el diagnóstico y la referencia del paciente suele ser tardío. La resección del neuroma y la reconstrucción con injerto nervioso tienen mejores resultados; otra alternativa quirúrgica es realizar transferencias nerviosas en presencia de avulsiones. El mejor pronóstico tiene lugar con un manejo oportuno, una exploración clínica cuidadosa y la individualización de las necesidades del paciente; estas decisiones complejas involucran a un equipo multidisciplinario, así como la difusión y educación para prevenir la lesión y realizar el diagnóstico oportuno.
\end{abstract}

Palabras clave: Lesión del plexo braquial, parálisis del plexo braquial congénito, lesión perinatal, parálisis superior de la extremidad, displasia glenohumeral.

\begin{abstract}
Actually, the surgical management of brachial plexus injuries is a great challenge. Despite the preventive measures applied by health systems, brachial plexus injuries continue to occur with and incidence of 0.38 to 5.1 per 1,000 live births worldwide and in Mexico from 0.37 to $0.87 \times 1,000,20 \%$ of these patients will have a residual neurological deficit, which will directly impact the function of the upper limb. The challenge of a surgical treatment is to bring the patient to the greatest possible functionality, without deteriorating the residual function that may exist. The surgical decision is determined by the surgeon considering the clinical manifestations, absence of neurological recovery over time, and surgical findings. Unfortunately, on many occasions the diagnosis and referral of the patient is usually late. Neuroma resection and nerve graft reconstruction have better results, another surgical alternative is to perform nerve transfers in the presence of avulsions. The best prognosis takes place with timely management, a careful clinical examination, and individualization of the patient's needs, these complex decisions involve a multidisciplinary team, as well as dissemination and education, to prevent injury, make the timely diagnosis and surgery needed at the right time.
\end{abstract}

Keywords: Brachial plexus injury, congenital brachial plexus palsy, perinatal injury, upper extremity palsy, glenohumeral dysplasia.

Correspondencia:

Marlene Vanessa Salcido Reyna

E-mail: drasalcidoreyna@gmail.com

Recibido: 09-02-2021. Aceptado: 10-02-2021.
Citar como: López AL, Pérez LFY, Salcido RMV. Lesión de plexo braquial obstétrico; una alternativa de manejo quirúrgico. Reporte de un caso y revisión de la literatura. Orthotips. 2021; 17 (1): 50-54. https://dx.doi. org/10.35366/99167 


\section{Introducción}

Las lesiones del plexo braquial tienen una incidencia de 0.38 a 5.1 por 1,000 nacimientos vivos a nivel mundial, en México es de 0.37 a 0.87 por 1,000 nacimientos vivos, sólo $46 \%$ de los pacientes tienen factores de riesgo identificables ${ }^{1}$ y $20 \%$ tendrán un déficit neurológico residual que impactará directamente la función de la extremidad superior. Las lesiones del plexo braquial aumentan los días de estancia hospitalaria en $20 \%$ y el costo directo hasta $40 \% .{ }^{1} \mathrm{La}$ exploración clínica permite un diagnóstico temprano, una clasificación de la lesión y una planeación quirúrgica adecuada. ${ }^{1}$

La clasificación es la siguiente: ${ }^{2}$

\section{Categoría I. Localización de la lesión}

1. Altas (involucra las raíces de C5-C6 con o sin extensión a C7).

2. Total/bajas (involucra todas las raíces).

\section{Categoría II. Tipo de lesión:}

1. Avulsión.

2. Ruptura.

La indicación quirúrgica debe ser entre el segundo y tercer mes de nacimiento en los siguientes casos: pacientes con lesiones totales del plexo braquial, con síndrome de Horner, con lesión alta, bíceps con

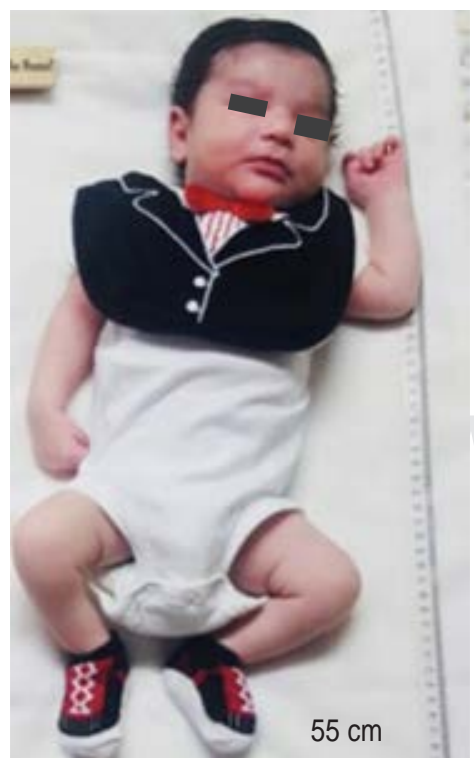

Figura 1:

Paciente recién nacido con peso de $3,700 \mathrm{~kg}$.

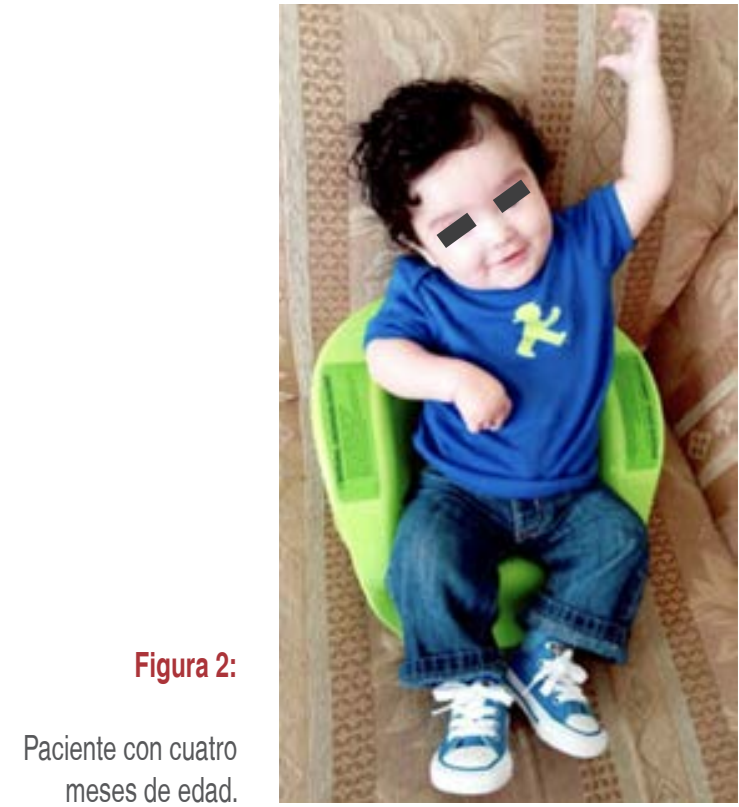

evaluación de cero en el examen de fuerza muscular y deltoides de cero.

Aquellos con lesión alta del plexo braquial que muestran alguna función del bíceps o deltoides deben tener seguimiento y reexaminación cada cuatro semanas, si al quinto mes el bíceps no está completamente normal, debe realizarse la cirugía. ${ }^{2}$

Los estudios complementarios como las radiografías sirven para evidenciar lesiones asociadas, tales como fractura de clavícula, primera costilla o húmero; sin embargo, carecen de un valor predictivo en la recuperación de la lesión nerviosa. La cirugía temprana ofrece ventajas funcionales y sensitivas, reduce la deformidad articular y disminuye las alteraciones del crecimiento asociadas a la lesión. ${ }^{3} \mathrm{El}$ abordaje quirúrgico inicia con la exploración del plexo braquial a través de una incisión supraclavicular para acceder a las raíces altas C5-C7 y supra-e infraclavicular para una lesión completa. La resección del neuroma y la reconstrucción con injerto del nervio sural sigue siendo el "estándar de oro»;2,3 aun así, la resección del neuroma cuando hay conducción es un tema controversial. Las transferencias nerviosas son una excelente opción cuando el extremo de la raíz no está disponible. La fuente proximal donante puede ser externa al plexo (extraplexal) o interna (intraplexal), se requiere un nervio receptor, la sutura puede ser directa o indirecta a través de un injerto nervioso. ${ }^{2}$ Posterior a la cirugía se realiza una inmovilización por tres semanas, es importante evaluar al paciente 
y evidenciar las escalas de movilidad cada tres meses los primeros dos años, cada seis meses hasta los cinco años y cada año hasta la adolescencia. ${ }^{2}$

\section{Presentación del caso}

Paciente masculino de cuatro meses de edad, diagnóstico de lesión de plexo braquial obstétrica. Fecha de nacimiento el 19 de marzo de 2016, producto de primera gesta de madre de 35 años con antecedente de hipotiroidismo, aumenta durante el embarazo $20 \mathrm{~kg}$, control prenatal, trabajo de parto de 14 horas, distocia de hombros con hipoxia neonatal, el paciente fue tratado en unidad de cuidados intensivos neonatal durante siete días. Flacidez completa de la extremidad torácica derecha. Peso: $3.700 \mathrm{~kg}$, talla: $55 \mathrm{~cm}$ (Figura 1); a los cuatro meses, en la exploración del paciente, se observó extremidad torácica derecha sin movilidad de hombro, codo con flexión del bíceps $M 3$, extensión de codo 0 , la muñeca no tiene movimiento de flexión ni extensión, flexión de dedos presente (Figura 2). Al quinto mes no presentó mejoría, bíceps M3 no funcional.

Se realizó intervención quirúrgica el día 29 de agosto de 2016, la cual consistió en exploración del plexo braquial con incisión supraclavicular, se encontró neuroma del tronco primario superior más avulsión de la raíz de $\mathrm{C} 7$, con integridad de las raíces de C8-T1. Se verificó la conducción nerviosa con neuroestimulador sin conducción del neuroma, por lo que éste se resecó y se reconstruyó con injerto del nervio sural, división anterior y posterior del tronco primario superior. Se
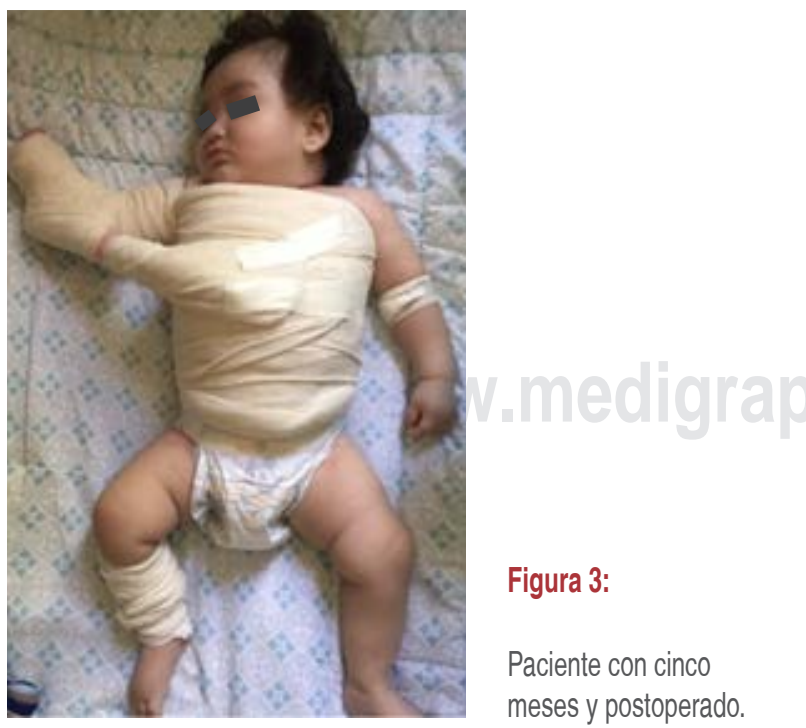

Figura 3:

Paciente con cinco meses y postoperado.

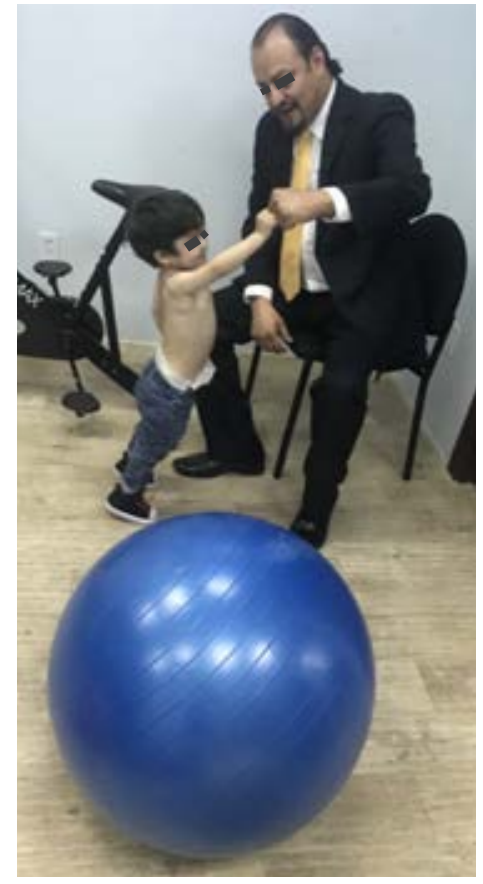

realizó transferencia de la rama distal del nervio espinal accesorio al nervio supraescapular y liberación anterior de hombro, coracoidectomía y alargamiento del pectoral mayor, reducción de la articulación glenohumeral, se colocó aparato de fibra de vidrio en rotación externa, centrando la articulación del hombro y corrigiendo la congruencia articular (Figura 3). Se envió a terapia física y se realiza seguimiento cada tres meses.

A los dos años de edad el hombro con abducción de 110 grados, flexión 110 grados, rotación externa 20 grados, codo flexión y extensión completa, mano con función completa y extensión completa del pulgar (Figura 4).

Paciente a los cuatro años de edad con abducción de 110 grados y rotación externa 10 grados que permite una actividad normal (Figura 5).

\section{Discusión}

En pacientes con lesión de plexo braquial obstétrico (PBO), la detección a través de la exploración es la base para tomar las decisiones quirúrgicas, en ausencia del bíceps funcional de los tres a seis meses, el paciente presenta un deterioro progresivo en las escalas de valoración funcional. ${ }^{4} \mathrm{Al}$-Qattan ${ }^{5}$ refiere esperar al cuarto mes para decidir el manejo quirúrgico. Este paciente presentó una recuperación de bíceps no funcional, por lo que se decidió manejo 
quirúrgico. La neurólisis de manera aislada que se hacía históricamente ya no está recomendada. ${ }^{6} \mathrm{Se}$ realizó en este paciente una exploración y reconstrucción de plexo braquial. La reconstrucción del plexo braquial permite una recuperación anatómica, funcional y sensitiva. ${ }^{7,8}$ El paciente tuvo una recuperación funcional de los movimientos de hombro tanto de ABD como de rotación externa; Bertelli ${ }^{9}$ describe mejores resultados con la reconstrucción de C5 con injerto hacia la división anterior del tronco primario superior más la transferencia del nervio espinal accesorio al nervio supraescapular atribuible a la reinervación de los músculos agonistas y antagonistas que estabilizan la articulación del hombro. En pacientes pediátricos la reconstrucción ofrece mejores resultados que en el adulto por la menor distancia, esto se debe a la menor longitud de las extremidades y a la mayor regeneración axonal. ${ }^{10}$ La liberación anterior aumenta el movimiento pasivo de la articulación y centra la cabeza humeral frente a la apófisis glenoidea, lo que lleva a la remodelación de las deformidades óseas y la corrección de la incongruencia de la articulación glenohumeral.

Los procedimientos microquirúrgicos, además de ser restauradores por derecho propio, aumentan la posibilidad de transferencias nerviosas como procedimientos secundarios y transferencias tendinosas que pueden mejorar aún más la funcionalidad. ${ }^{11}$

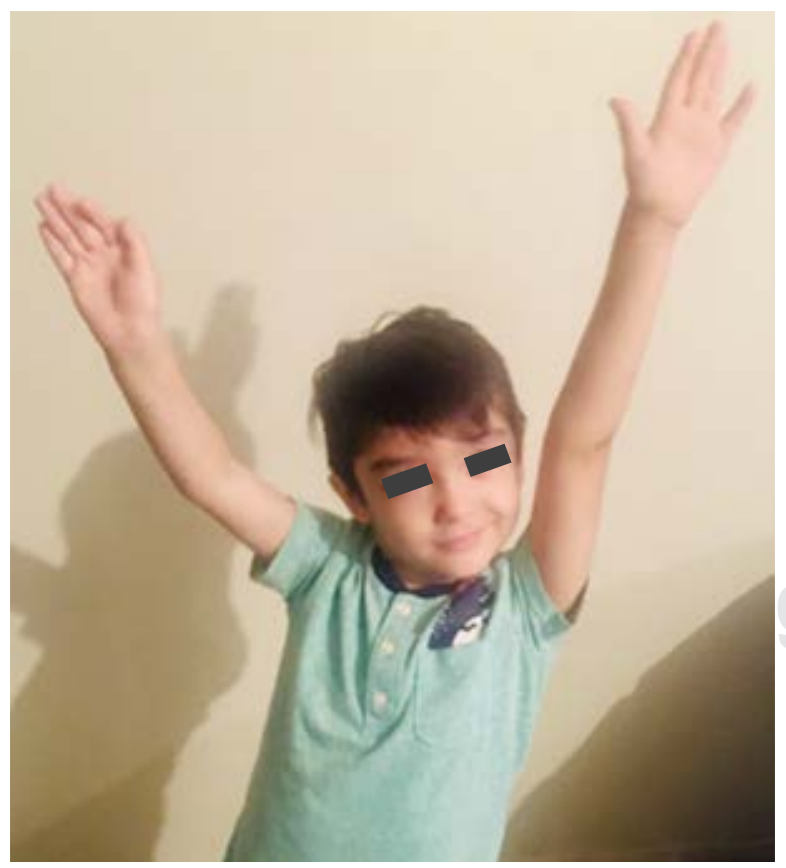

Figura 5: Paciente a los cuatro años de edad.
Las guías canadienses ${ }^{12}$ hacen hincapié en el equipo multidisciplinario, refieren que el tratamiento es efectivo si los especialistas locales están involucrados desde la difusión y referencia, por lo tanto, se busca a través de grupos multidisciplinarios, conectar la educación y consciencia pública para modificar factores de riesgo, derivación, evaluación y terapia.

La resección del neuroma y reconstrucción con injerto de nervios son el estándar de atención microquirúrgica para las lesiones por rotura con las que deben compararse otras técnicas. ${ }^{13}$ El tratamiento primario, es decir, la restauración de la función nerviosa, debe realizarse entre los tres y seis meses de edad y recibir máxima prioridad. ${ }^{14}$

Existe un declive en la incidencia de la enfermedad entre 1997 y 2012:15 sin embargo, la alta morbilidad de la lesión hace prioritario continuar con trabajos de investigación que favorezcan la identificación de factores de riesgo prevenibles, así como ventajas del manejo en tiempos adecuados. El objetivo es transformar la atención en un modelo que reconoce las necesidades del paciente, desde el parto hasta la madurez completa. ${ }^{16}$

\section{Conclusiones}

El tratamiento inicial debe ser conservador, el tema de controversia es el momento en el que debe comenzar la intervención y específicamente cuando sí se justifica la cirugía. ${ }^{11}$ El manejo de las lesiones de PBO se ha modificado en los últimos 25 años con el advenimiento de la microcirugía. ${ }^{17}$ La decisión de una intervención quirúrgica es un equilibrio entre intervenir a tiempo y permitir la recuperación espontánea. Los avances en la microcirugía han brindado alternativas para restaurar el movimiento y la función, demostrando además ser un procedimiento seguro; sin embargo, la cirugía está infrautilizada, ya que se llega a creer "que todas las parálisis neonatales de plexo se recuperan y hay que esperar un año»; es deseable una derivación inmediata durante el primer mes de vida. ${ }^{17}$

La reconstrucción óptima necesita considerar un plan tanto a corto como a largo plazo, la reinervación máxima puede involucrar al injerto nervioso como a las transferencias nerviosas. El pronóstico depende de tres factores: la severidad de la lesión, la velocidad de la recuperación y la calidad del manejo. ${ }^{2}$ Además, el conocimiento de la lesión y de la historia natural de la lesión del plexo braquial obstétrico permite establecer en cada paciente una estrategia única. Se busca 
siempre la reconstrucción con injerto con el objetivo de aprovechar una mayor cantidad de axones y una reconstrucción anatómicamente correcta.

A pesar de las ventajas de las transferencias nerviosas la morbilidad asociada a largo plazo aún no está clara. La disfunción directa del donante en una extremidad ya comprometida puede tener implicaciones y el efecto de la denervación parcial en el crecimiento musculoesquelético es una preocupación. Siempre la ventaja de la reconstrucción con injerto es la recuperación sensitiva, y la transferencia nerviosa podrá utilizarse como cirugía secundaria para mejorar la función lograda o en una reinervación fallida. ${ }^{3,18}$

Por otra parte, la cirugía no debe retrasarse porque las deficiencias asociadas son mucho más significantes (deformidad articular, pérdida del crecimiento, contractura severa, falta de movilidad); una de las ventajas de la cirugía temprana es la disminución del tiempo de denervación muscular y, por lo tanto, menor alteración muscular y articular, los circuitos de reconocimiento de la extremidad por el cerebro son menos afectados, así como mayor tolerancia del paciente a la inmovilización y el uso de aparatos de yeso. ${ }^{3}$

Las claves para el mejor pronóstico van más allá de la microcirugía, involucran a un equipo multidisciplinario que participe en la difusión y educación para prevenir la lesión, hacer la referencia en tiempo correcto y dar el tratamiento adecuado; esto involucra no sólo a médicos, sino también a terapistas físicos, enfermería, trabajadores sociales, médicos de primer nivel y a toda la población. ${ }^{19}$

\section{Referencias}

1. Buterbaugh KL, Shah AS. The natural history and management of brachial plexus birth palsy. Curr Rev Musculoskelet Med. 2016; 9: 418-426.

2. Borrero JL. Surgical technique in brachial plexus injuries. Martin Dunitz, Condon. 2000; 189-204.

3. Gilbert A, Whitaker I. Obstetrical brachial plexus lesions. Journal of Hand Surgery. 1991; 16 (5): 489-491.

4. Abid A. Brachial plexus birth palsy: management during the first year of life. Orthop Traumatol Surg Res. 2016; 102 (1): S125-S132.

5. Al-Qattan MM, El-Sayed AAF. The outcome of primary brachial plexus reconstruction in extended Erb's obstetric palsy when only one root is available for intraplexus neurotization. Eur $\mathrm{J}$ Plast Surg. 2017; 40 (4): 323-328.

6. Andrisevic E, Taniguchi M, Partington MD, Agel J, Van Heest AE. Neurolysis alone as the treatment for neuroma-in-continuity with more than $50 \%$ conduction in infants with upper trunk brachial plexus birth palsy: clinical article. J Neurosurg Pediatr. 2014; 13 (2): 229-237.

7. Argenta AE, Brooker J, Maclssac Z, Natali M, Greene S, Stanger M, et al. Obstetrical brachial plexus palsy: can excision of upper trunk neuroma and nerve grafting improve function in babies with adequate elbow flexion at nine months of age? $\mathrm{J}$ Plast Reconstr Aesthet Surg. 2016, 69 (5): 629-633.

8. Bahm J, Becker M, Disselhorst-Klug C, Williams S, Meinecke $\mathrm{L}$, Müller $\mathrm{H}$, et al. Surgical strategy in obstetric brachial plexus palsy: the Aachen experience. Semin Plast Surg. 2004; 18 (04): 285-299.

9. Bertelli JA, Ghizoni MF. Nerve root grafting and distal nerve transfers for c5-c6 brachial plexus injuries. J Hand Surg Am. 2010; 35 (5): 769-775.

10. Bertelli JA, Ghizoni MF, Little KJ, Zlotolow DA, Soldado F, Cornwall R. Examination, C.. nerve transfers in birth related brachial plexus injuries. Where do we stand? Journal of Hand Surgery. 2016; 32 (2): 175-190.

11. Kwazneski DR, lyer RC, Panthaki Z, Armstrong MB. Controversies in the diagnosis and treatment of pediatric brachial plexus injuries. Journal of Craniofacial Surgery. 2009; 20 (4): 1036-1038.

12. Coroneos CJ, Voineskos SH, Christakis MK, Thoma A, Bain JR, Brouwers MC, et al. Obstetrical brachial plexus injury (OBPI): Canada's national clinical practice guideline. BMJ Open. 2017; 7: e014141.

13. Hale HB, Bae DS, Waters PM. Current concepts in the management of brachial plexus birth palsy. J Hand Surg. 2010; 35 (2): 322-331.

14. Hierner R, Becker M, Berger A. Indications and results of operative treatment in birth-related brachial plexus injuries. Handchirur Mikrochir Plast Chir. 2005; 37 (5): 323-331.

15. Defrancesco CJ, Shah DK, Rogers BH, Shah AS. The epidemiology of brachial plexus birth palsy in the United States: declining incidence and evolving risk factors. J Pediatr Orthop. 2019; 39 (2): e134-e140.

16. Bertelli JA, Ghizoni MF, Soldado F. Patterns of brachial plexus stretch palsy in a prospective series of 565 surgically treated patients. J Hand Surg Am. 2017; 42 (6): 443-446.e2.

17. Smith BW, Daunter AK, Yang LJS, Wilson TJ. An update on the management of neonatal brachial plexus palsy-replacing old paradigms a review. JAMA Pediatr. 2018; 172 (6): 585-591.

18. Hoang D, Chen VW, Seruya M. Recovery of elbow flexion after nerve reconstruction versus free functional muscle transfer for late, traumatic brachial plexus palsy: a systematic review. Plast Reconstr Surg. 2018; 141 (4): 949-959.

19. Heise CO, Siqueira MG, Martins RS, Foroni LH, Sterman-Neto $\mathrm{H}$. Distal nerve transfer versus supraclavicular nerve grafting: comparison of elbow flexion outcome in neonatal brachial plexus palsy with $\mathrm{C} 5-\mathrm{C} 7$ involvement. Childs Nerv Syst. 2017; 33 (9): 1571-1574. 